\title{
Tresca criterion for plasticity and viscoplasticity: Application to localization in biaxial loading conditions
}

\author{
J.L. Dequiedt, C. Bolis, and F. Dambakizi \\ CEA, DAM, DIF, 91297 Arpajon, France
}

\begin{abstract}
It has been proved that plastic instabilities in biaxial stretching conditions were better reproduced by using a Tresca yield surface rather than a Von Mises one. The simulation of the phenomenon in an expanding TA6V4 (Ti-6Al-4V alloy) shell experiment is performed using the Tresca criterion and both elasto-plastic and viscoplastic constitutive models: in this aim, Tresca flow surfaces had to be defined in viscoplasticity. The two models exhibit localization but, whereas the elastoplastic case develops shear banding in times in agreement with the onset of instabilities in the experiment, the viscoplastic case develops diffuse necking at later times. On the contrary, the viscoplastic simulation exhibits patterns the size of which seems in better agreement with the experimental ones.
\end{abstract}

\section{Introduction}

The ductility of shells in expansion is limited by the localization of deformation in necks or shear bands which leads to the fragmentation of the shell. The phenomenon appears in either uniaxial or biaxial stretching encountered in expanding cylinder or expanding sphere experiments respectively, for instance (Olive et al. [1], Mercier et al. [2]).

It is usually explained at the macroscopic scale by instabilities of the plastic deformation of the shell coming from the competition between hardening of the material by defect storage and thermal softening due to temperature increase in dynamic loading conditions (Jouve [3]).

In biaxial stretching, localization is also strongly dependent on the shape of the plasticity yield surface and it was proved that limits to ductility in agreement with experimental observations could be found by using angular plasticity surfaces (Storen and Rice [4] for instance) rather than smooth surfaces. Angular surfaces are physically justified by polycrystal plasticity simulations which exhibit the formation of vertices on the yield surface after some amount of deformation (Toth et al. [5]): as crystallographic texture forms, there are states of stress for which plastic slips of different orientations can be activated.

One simple way to reproduce the existence of a vertex in biaxial stretching is to use the Tresca yield surface rather than the Von Mises one (Dequiedt, [6]).

However, viscosity, and more generally sensitivity to plastic strain rate, should not be neglected since the strain rates reached in expanding shell experiments are generally over 1000/s. So, it is necessary to extend the Tresca criterion to viscoplastic models more appropriate for the material behaviour in such conditions.

The Tresca approach is developed in the following for an expanding shell experiment on titanium alloy TA6V4: the development of localization is simulated with both a Steinberg-Cochran-Guinan (SCG) elasto-plastic model [7] and a Preston-Tonks-Wallace (PTW) viscoplastic model [8].

In section 2, an extension of the Tresca criterion to viscoplasticity is defined in this purpose. In Section 3, the expanding shell experiment is described and the experimental results are analysed; then, local simulations are carried on in order to reproduce the development of localization in the shell; the SCG and PTW results are compared with experimental observations.

\section{Tresca criterion in plasticity and viscoplasticity}

In the case of elasto-plastic materials, the constitutive model defines an elastic limit as a function of the equivalent plastic strain $\sigma_{Y}\left(\varepsilon^{p}\right)$ (and eventually, temperature, pressure,...).

For the Von Mises criterion, the stress state remains inside an elastic domain given by the equation:

$$
F(\sigma)=\sigma^{\dot{e} q}-\sigma_{Y}\left(\varepsilon^{p}\right) \leq 0
$$

In equation (1), $\sigma^{\dot{e} q}$ is the equivalent stress $\left(\sigma^{\dot{e} q}=\right.$ $\sqrt{3(\mathbf{s}: \mathbf{s}) / 2})$ where $\mathbf{s}$ is the deviatoric stress.

The material plastifies when it reaches the frontier of the domain which is called the yield surface; in the Von Mises case, this surface is regular. The plastic strain rate is normal to the yield surface and its intensity (given by the plastic multiplier $\dot{\lambda}$ ) is independent of the stress:

$$
\dot{\boldsymbol{\varepsilon}}^{\mathbf{p}}=\dot{\lambda} \frac{\partial F}{\partial \sigma}
$$

For the Tresca criterion, $F(\sigma)$ is a function of the two extremes of the principal stresses:

$$
F(\sigma)=\operatorname{Max}\left(\sigma_{i}-\sigma_{j}\right)-\sigma_{Y}\left(\varepsilon^{p}\right)
$$

The yield surface possesses vertex points corresponding to cases when two of the principle stresses are equal. In these points the normal is not unique which gives an additive degree of freedom to the plastic strain rate; this is favourable to the development of instability modes.

For both criteria, we chose to keep the Von Mises definition of the equivalent plastic strain as a strain hardening parameter; its time evolution is thus given by:

$$
\dot{\varepsilon}^{p}=\sqrt{\frac{2}{3} \dot{\varepsilon}^{\mathbf{p}}: \dot{\boldsymbol{\varepsilon}}^{\mathbf{p}}}
$$




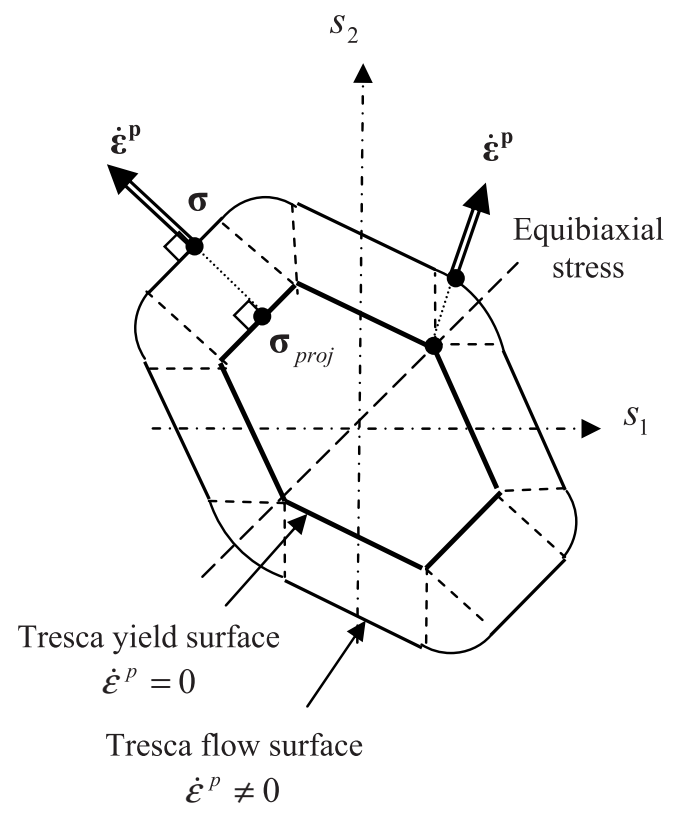

Fig. 1. Tresca criterion in viscoplasticity - flow surfaces and plastic strain rates.

In viscoplasticity, the constitutive model defines a flow stress as a function of the plastic strain and of the plastic strain rate: $\sigma_{Y}\left(\varepsilon^{p}, \dot{\varepsilon}^{p}\right)$. The elastic limit corresponds to $\sigma_{Y}\left(\varepsilon^{p}, \dot{\varepsilon}^{p}=0\right)$. The material plastifies when the stress state is outside the elastic domain.

In the Von Mises case, the plastic strain rate satisfies $\sigma^{\dot{e} q}-\sigma_{Y}\left(\varepsilon^{p}, \dot{\varepsilon}^{p}\right)=0$ and $\dot{\boldsymbol{\varepsilon}}^{\mathbf{p}}$ is still normal to the yield surface. The flow surfaces, i.e. surfaces of homogeneous plastic strain rate, are thus homothetic to the yield surface.

We chose to extend viscoplasticity to the Tresca criterion in the following way; we defined Tresca flow surfaces as surfaces of equal distance to the Tresca yield surface:

$$
\delta(\sigma)=\sigma_{Y}\left(T, \varepsilon^{p^{e q}}, \dot{\varepsilon}^{p^{e q}}\right)-\sigma_{Y}\left(T, \varepsilon^{p^{e q}}, \dot{\varepsilon}^{p^{e q}}=0\right)
$$

The distance in the stress space is taken "in the Von Mises sense", with $\sigma_{\text {proj }}$ being the projection of stress on the Tresca yield surface:

$$
\delta(\sigma)=\sqrt{\frac{3}{2}\left(\sigma-\sigma_{p r o j}\right):\left(\sigma-\sigma_{p r o j}\right)}
$$

The Tresca flow surfaces and the strain rate tensors are represented on figure 1 in the plane of the first principal components $\left(s_{1}, s_{2}\right)$ of the deviatoric stress $\mathbf{s}$.

Let us remark that the viscoplasticity regularizes the corners of the Tresca surface: in other words, the degree of freedom on the glide direction given by the vertex in plasticity is removed by viscosity and the plastic strain rate is unique for a given stress. This can be justified from the physical point of view by admitting that, when several slip systems are activated, the strain rate due to each of them is fixed, as a function of its own viscosity.

However, when the sensitivity to plastic strain rate is not too high, the flow surfaces have strong curvature points in front of the vertices of the yield surface.

$$
s_{2} \dot{\boldsymbol{\varepsilon}}^{\mathbf{p}} \sigma \sigma_{\text {proj }} \dot{\varepsilon}^{p}=0 \dot{\varepsilon}^{p} \neq 0 \dot{\boldsymbol{\varepsilon}}^{\mathbf{p}} s_{1}
$$

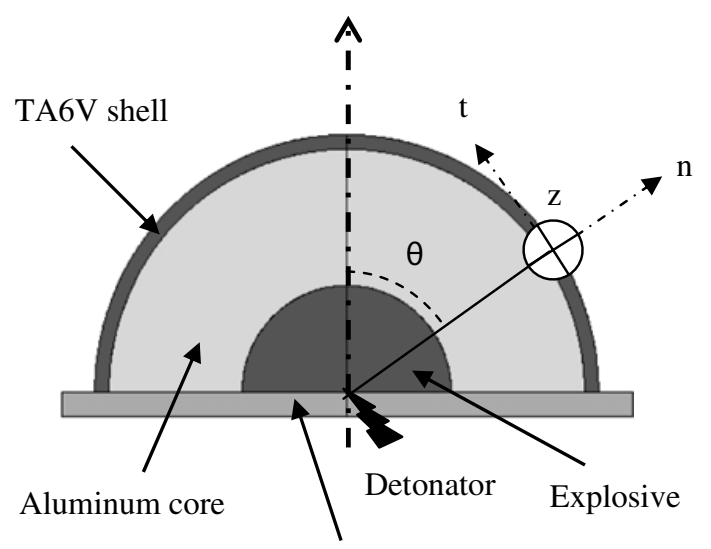

Steel bottom

Fig. 2. Expanding hemisphere set-up.

\section{Hemisphere expansion experiment}

\subsection{Experimental results and global simulation of the experiment}

The experimental set-up has been used for different materials in the past: it is schematized on figure 2. A shock wave generated by an explosive sphere is transmitted to a hemispherical shell of TA6V4 through an aluminum core. The shell just lies on a steel bottom and separates from the core little time after the arrival of the detonation wave on the free surface. Then, it deforms during a free flight expansion.

- Various diagnostics were performed during the test:

- Velocity measurements which revealed the absence of spalling at the shock arise;

- High speed camera images and laser images [9] which allowed following the localization and fragmentation process in the shell;

- Streak camera record which confirm the time of cracking.

According to the streak camera, the first instabilities are visible at time $\mathrm{t} \approx 24 \mu$ s and the first fractures at time $\mathrm{t} \approx 27 \mu \mathrm{s}$. These times are confirmed by the laser images showing a rather homogeneous localization process on the whole hemisphere (figure 3). The cracks have a roughly isotropic distribution of orientations except near the bottom where cracks oriented along the meridians prevail. An average radius of $6.4 \mathrm{~mm}$ for the fragments was evaluated from processing of the image.

The expansion of the shell is simulated in a lagrangian hydrodynamic code in axisymmetric geometry. The initial shock reaches the TA6V4 shell at $\mathrm{t} \approx 15 \mu \mathrm{s}$. The components $D_{t t}$ and $D_{z z}$ of the strain rate tensor in the local coordinate system (as defined on figure 2) are plotted as functions of the angular position for different times on figure 4. Up to the time of fragmentation, the major part of the shell is loaded in nearly homogeneous equi-biaxial stretching which explains why the localization patterns are the same all over the shell. The close neighborhood of the bottom is affected by release waves but which do not have time to propagate through a long distance until fragmentation. 


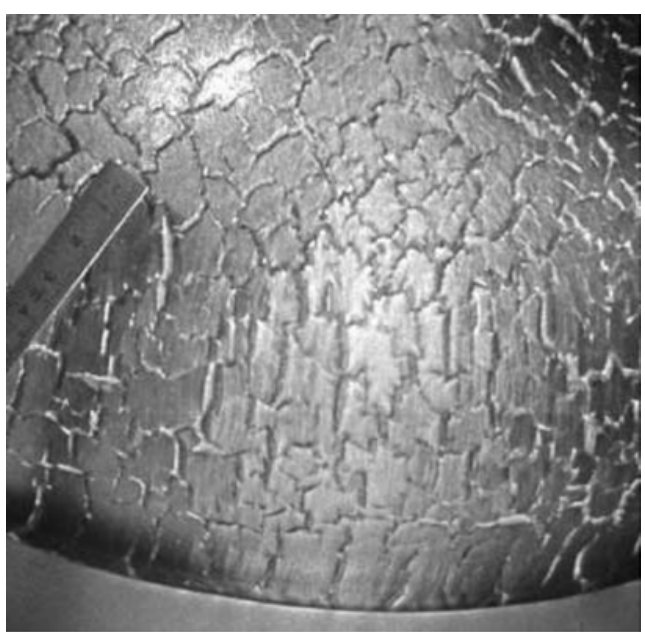

Fig. 3. Laser image of the hemisphere with localization pattern visible.

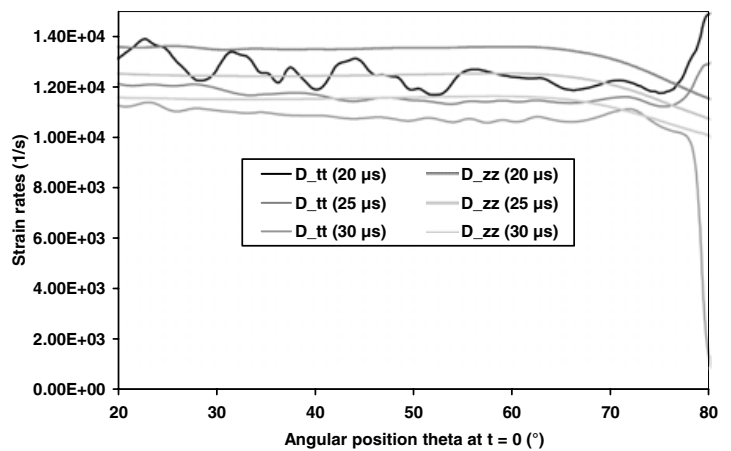

Fig. 4. Components of the strain rate tensor in the TA6V4 shell at different times.

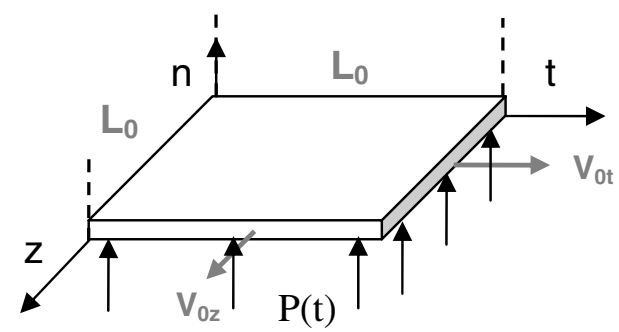

Fig. 5. Geometry and conditions of the local simulation.

\subsection{Simulation of localization}

Simulations of localization are performed by representing a small part of the shell as a plane plate loaded in equibiaxial tension and submitted on its lower surface to a uniform pressure impulse $P(t)$ (figure 5). Constant velocities $V_{0 t}$ and $V_{0 z}$ are imposed on the boundaries in such a way that the initial strain rates are equal to the ones estimated at $\mathrm{t}=20 \mu \mathrm{s}$ of the expansion $\left(D_{t t} \approx D_{z z}=1.410^{4} \mathrm{~s}^{-1}\right)$. Pressure $P(t)$ is a piecewise linear fit of the pressure applied inside the shell during the propagation of the detonation wave (figure 6). Localization is displayed in such simulations provided that a small "noise" is introduced: we chose to add a small spatial fluctuation to the elastic limit.

Two simulations are performed with the Tresca criterion and the SCG and PTW plasticity models respectively.

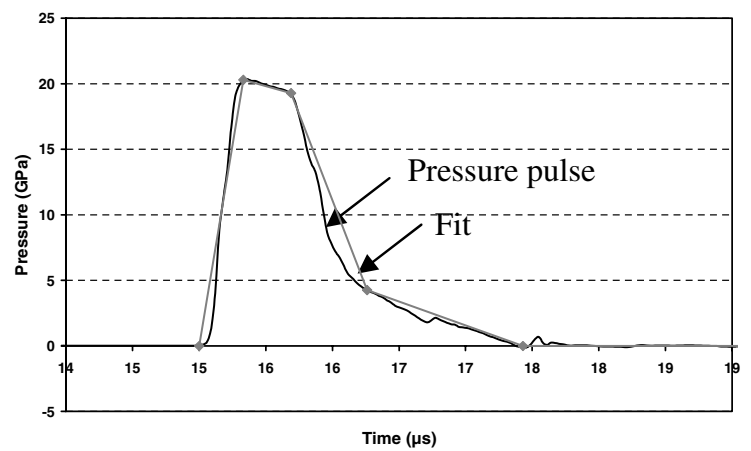

Fig. 6. Internal pressure in the shell experiment and fit for the local simulation.

The associated results are given on figures 7 and 8: maps of equivalent plastic strain in average plane and cross-section are shown at different times.

The shock and release waves generated by the pressure pulse induce a large amount of plastic strain which is inhomogeneous in the thickness but tends to saturate the yield stress; so, conditions are fulfilled for the emergence of instabilities.

The SCG simulation exhibits a localization process starting at short time (but after the inner pressure has fallen to zero): a network of shear bands appears which produces a sort of "orange skin effect" in the average plane. The bands cross the whole thickness and there is no real evidence whether they initiate in the lower or upper face or inside the plate. Latter, the deformation concentrates in some of these bands forming patterns of about $2 \mathrm{~mm}$ in the average plane. The phenomenon is hardly noticeable in the simulation at time $\mathrm{t} \approx 25 \mu$ s while significant texture is visible in the experiment at time $\mathrm{t}=24 \mu \mathrm{s}$ : so, the simulation slightly underestimates the rate of growth of instabilities but the characteristic time scale is relevant.

In the PTW simulation, localization develops much more slowly: after some amount of extension, necking appears in the plate. The necks are very large at the beginning and latter shrink as they concentrate the deformation; they form patterns of approximate length scale $6 \mathrm{~mm}$ in rather good agreement with the fragment size identified on the images.

\section{Conclusion}

The capacity of the Tresca criterion with both elastoplastic and viscoplastic constitutive models to reproduce localization in biaxial stretching and high strain rates loading conditions was evaluated in this work. In this aim, an expanding hemisphere experiment performed on TA6V4 alloy was analyzed and the localization process was simulated.

With the elasto-plastic SCG model, the use of the Tresca criterion instead of the Von Mises one introduces a vertex point on the yield surface in equi-biaxial tension which leads to a quick development of a network of shear bands: the phenomenon develops in time scales comparable to the ones identified experimentally. However, the 


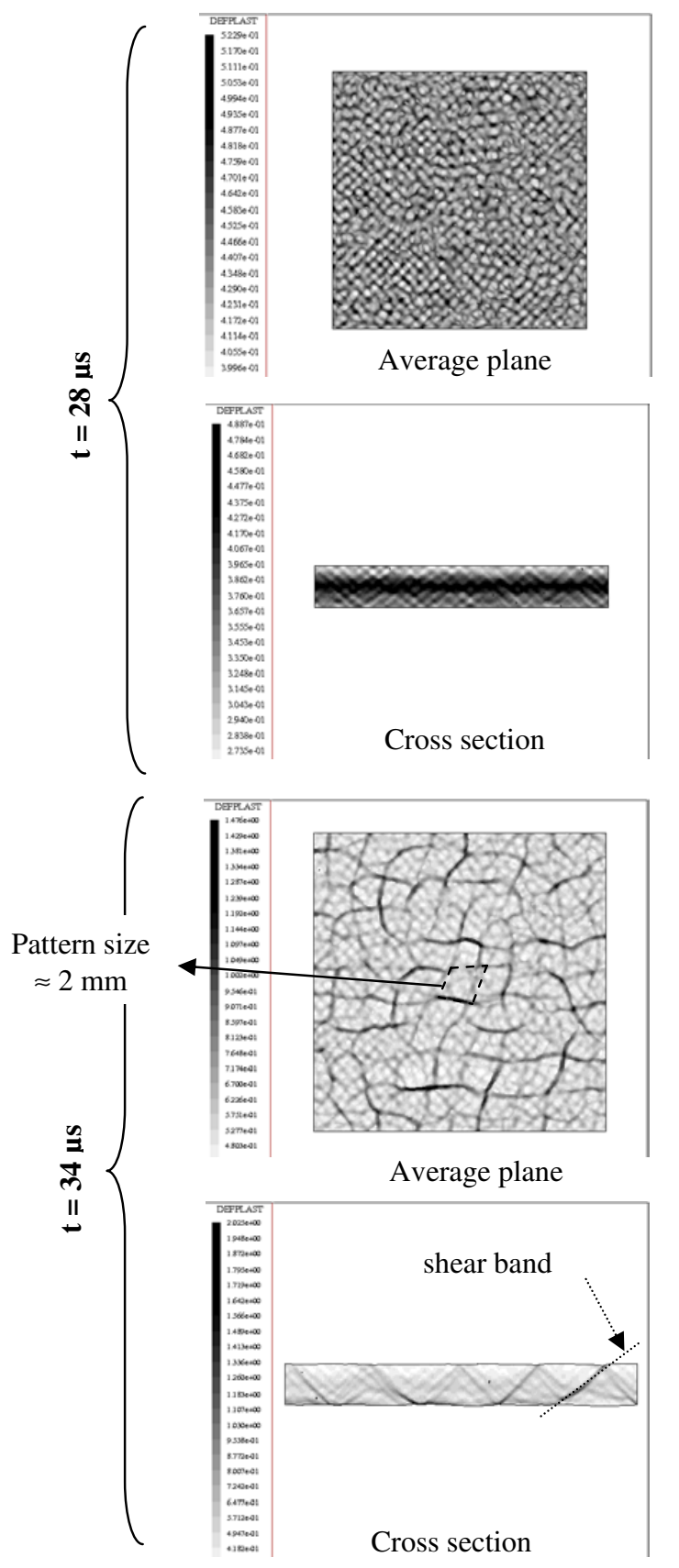

Fig. 7. Maps of equivalent plastic strain in average plane and cross section in the TA6V4 SCG simulation at different times.

localization pattern is smaller than the one observed on the camera images.

For the viscoplastic PTW model, which is a priori more suitable for high strain rate conditions, diffuse necking develops with a length scale in agreement with the one observed on camera images but with a characteristic time much longer than the experimental one. The plastic strain rate dependence does not only limit the rate of growth of instabilities but also smoothes the vertices of the Tresca surface: this prevents the homogeneous deformation to bifurcate into a localized one.

It seems that the coupling of viscoplastic effects with the evolution of the shape of the yield surfaces and the formation of vertices needs to be evaluated and modeled in a
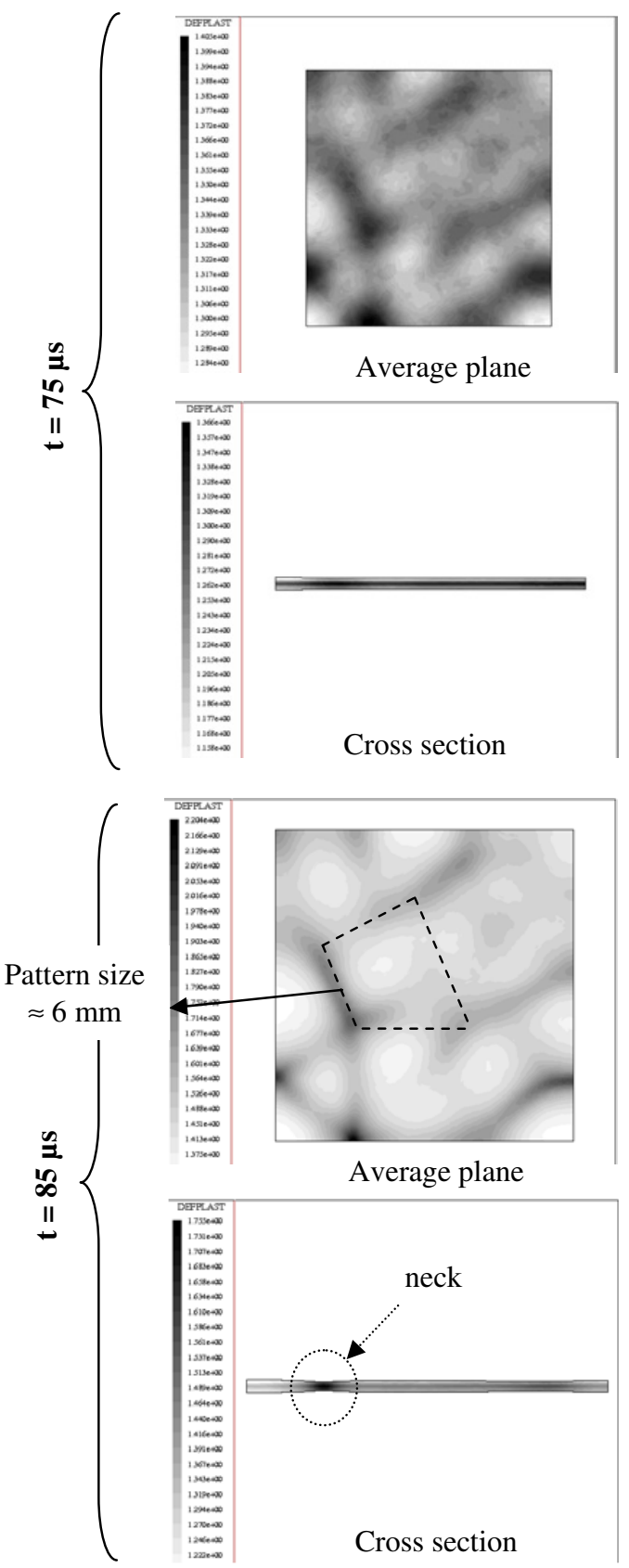

Fig. 8. Maps of equivalent plastic strain in average plane and cross section in the TA6V4 PTW simulation at different times.

more precise manner: among others, polycrystal plasticity simulations integrating plastic strain rate dependence may be helpful.

\section{References}

1. F. Olive, A. Nicaud, J. Marilleau, R. Loichot. Rupture behaviour of metals in explosive expansion. In: Harding, J. (Eds) Second Conference on the Mechanical Properties of Materials at High Rates of Strain, Institute of Physics, (Oxford 1979), 242-251.

2. S. Mercier, N. Granier, A. Molinari, F. Llorca, F. Buy. Multiple necking during the dynamic expansion 
of hemispherical metallic shells from experiments to modelling. Journal of the Mechanics and Physics of Solids, 58,7 (2010), 955-982.

3. D. Jouve. Dynamic plastic necking instabilities for a biaxially pulled plate. In: $8^{\text {th }}$ International DYMAT Conference, (Dijon 2006), 251-256.

4. S. Storen, J.R. Rice. Localized necking in thin sheets. Journal of the Mechanics and Physics of Solids, 23 (2010), 421-441.

5. L.S. Toth, D. Dudzinski, A. Molinari. Forming limit predictions with the perturbation method using stress potential functions of polycrystal viscoplasticity. International Journal of Mechanical Science, 38 (1996), 805-824.
6. J.L. Dequiedt. Localization in elasto-plastic materials: Influence of the plasticity yield surface in biaxial loading conditions. International Journal of Solids and Structures, 47 (2010), 2937-2951.

7. D.J. Steinberg, S.G. Cochran, M.W. Guinan. A constitutive model for metals applicable at high-strain rate. Journal of Applied Physics, 51, 3 (1980), 1498-1504.

8. D. Preston, D. Tonks, D. Wallace. Model of plastic deformation for extreme loading conditions. Journal of Applied Physics, 93 (2003), 211-220.

9. P.A. Frugier. Laser Imaging: Picturing high-explosive driven experiments with nanosecond to picosecond exposure times. 29th International Congress on High Speed Imaging and Photonics (Iwate, Japan 2010). 